\title{
TUNABLE FERROELECTRIC COMPONENTS IN LTCC TECHNOLOGY
}

\author{
A. Deleniv*, T.Hu ${ }^{\&}$, H.Jantunnen ${ }^{\&}$, S. Gevorgian*,\# \\ * Department of Microelectronics, Chalmers University of Technology \\ 41296 Gothenburg, Sweden: anatoli@ep.chalmers.se, spartak@ep.chalmers.se \\ \# Microwave and High Speed Research Center, Ericsson AB \\ 43184 Moelndal, Sweden \\ \& University of Oulu, Microelectronics and Materials Physics Laboratory and EMPART \\ Research Group of Infotech Oulu, P. O. BOX 4500, FIN-90014 Oulu, Finland \\ hutao@ees2.oulu.fi, heja@ees2.oulu.fi
}

\begin{abstract}
Designs issues of a tunable matching network and a tunable phase shifter based on LTCC ferroelectric technology are discussed. Two devices are designed for operation in $K$ frequency band. Test structure measurements are presented along with extracted parameters of used ferroelectrics (dielectric permittivity and tunability). Simulated and experimental performances are presented.
\end{abstract}

\section{INTRODUCTION}

Low Temperature Cofired Ceramics (LTCC) technology is widely used to fabricate cost effective and reliable microwave components and devices [1-3]. Today LTCC devices include passive microwave components, such as transmission lines, capacitors, filters etc. The active components based on semiconductors (transistors, varactors ICs) can be used only by assembling them on the surface as discrete components [4]. Incorporation of ceramics with tunable dielectric properties will enhance the possibilities of LTCC technology, enabling monolithic integration of components such as varactors, filters, phase shifters etc.

In this work we discuss design issues of two tunable components based on ferroelectric LTCC. To design these components we have fabricated and characterized experimentally novel ferroelectric films compatible with LTCC processes. The measured ceramic film parameters are used as input data in simulations of matching networks and power splitters. The evolution of these devices, starting from simple coplanar design to more complex two layered structures is presented. The tuneability of components with coplanar and two-layer configuration and their simplified modeling are discussed.

\section{CHARACTERIZATION OF FERROELECTRIC LTCC FILMS}

Special test structures are designed and fabricated for microwave measurements cermics film parameters. In test structures, and, actual devices, two main limitations are taken into account: i) The minimum size features of the screen printing process used for conductive strips is $200 \mu \mathrm{m}$, and ii) The required DC voltages are small. Based on these limitations we developed a test structure and measurement setup for microwave measurements of dielectric properties of tuneable ferroelectric LTCC films, Fig. 1. The cross section of the test structure is shown in Fig. 2, where the ferreoelctric films are sandwiched between the bottom electrode and two top conductive strips.

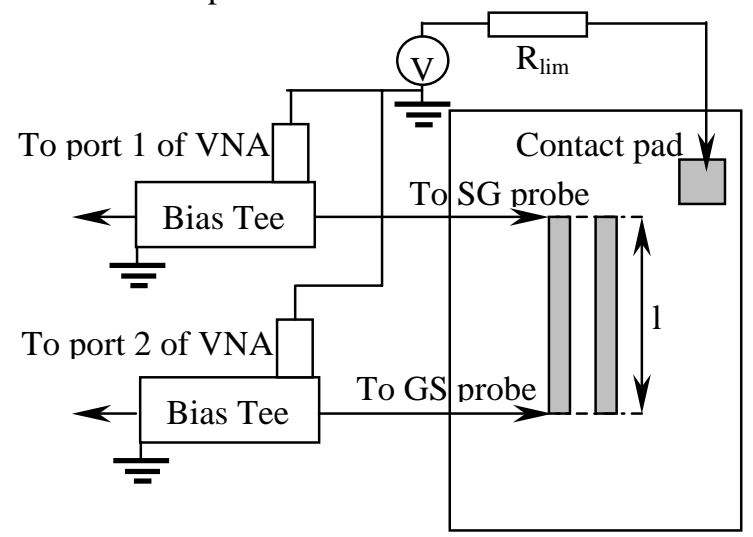

Fig.1 Layout of the test structure and measurement set-up

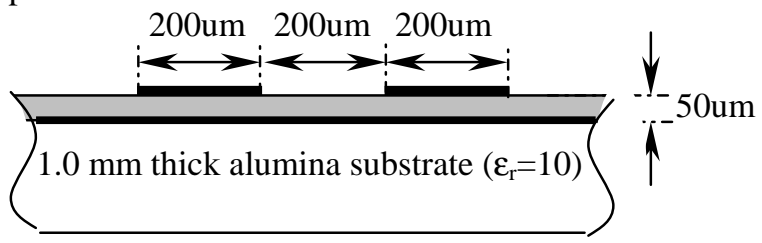

Fig. 2 Cross-section of the test structure.

It is well known, that two coupled lines support two modes (odd and even) with electric and magnetic walls in the symmetry plane. 
For on wafer microprobe measurements (using Signal-Ground (SG) $400 \mu \mathrm{m}$ pitchsize microprobes) only odd mode is excited. For the designed set-up, Fig.1, both probe tips are under the same DC potential, hence no DC field is applied between them. The high voltage is applied to the ground metallization using a via to the ground plane (contact pad, Fig.1) beneath ferroelectric film. A large current limiting resistor is connected in series with DC voltage source.

For specified sizes of the test structure dielectric permittivity of the ferroelectric film is computed form measurements of propagation constant of the odd mode. The discontinuity of the open ends in the test structure is taken into acount using measurements of two test structures with different lengths, $2.4 \mathrm{~mm}$ and $3.2 \mathrm{~mm}$. A typical data for extracted dielectric constant are presented in Fig. 3 for $100 \mathrm{~V}$ and $200 \mathrm{~V}$ applied voltages, corresponding to $2 \mathrm{~V} / \mu \mathrm{m}$ and $4 \mathrm{~V} / \mu \mathrm{m}$ DC field. As it can be seen the tunability is about $17 \%$ over entire frequency band.

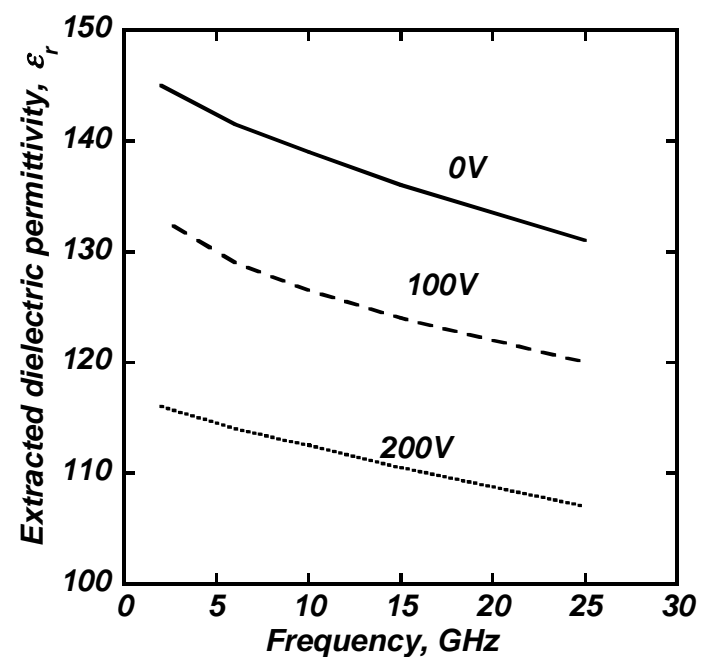

Fig.3 Extracted dielectric permittivity.

\section{DESIGN AND PERFORMANCE OF DEVICES: COPLANAR-PLATE VERSION}

\subsection{Phase shifter}

A unit cell of a loaded line phase shifter is shown in Fig.4a. For example under discussion we choose $Z \boldsymbol{c c}=56.5 \mathrm{Ohm}, \theta=\pi / 6$ and $\boldsymbol{C}(0)=0.19 \mathrm{pF}$. Such a unit cell produces about $8 \mathrm{deg}$. phase shift assuming $\sim 17 \%$ tunability in the capacitance. It follows from the above measurements that at least 12 cells need to be cascaded to obtain $90^{\circ}$ phase shift. The final layout of the developed phase shifter $(\mathrm{f}=26 \mathrm{GHz})$, including quarter-wavelength input/output matching (25Ohm image impedance to standard $50 \mathrm{Ohm}$ input level) sections is shown in Fig.4b. a)

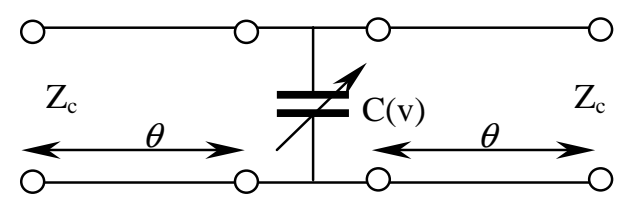

b)

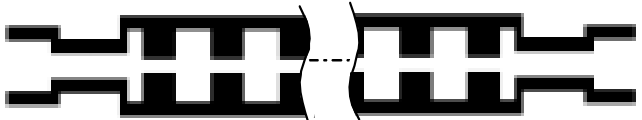

Fig.4 Unit cell (a) and layout of the phase shifter (b).

\subsection{Tunable matching network}

The equivalent circuit of a double stub matching network is shown in Fig.5. The complex load $\bar{Z}$ load $=\mathbf{1} \pm \mathbf{0 . 1}(\boldsymbol{1}+\boldsymbol{j})$ can be matched with a proper choice of electrical length $\boldsymbol{\theta}$ and their input reactance's $\boldsymbol{Z i n} \mathbf{1}$ and $\boldsymbol{Z i n} \boldsymbol{2}$ respectively. It can be shown that $\theta=\pi / 4$ results in lowest stub input reactance's range needed. The slot between the strips is small in low impedance stubs, while the strip separation in the main line is much larger. Thus, the DC field applied between the strips will tune the electric length and characteristic impedance only for stubs, while no changes occur in the main line.

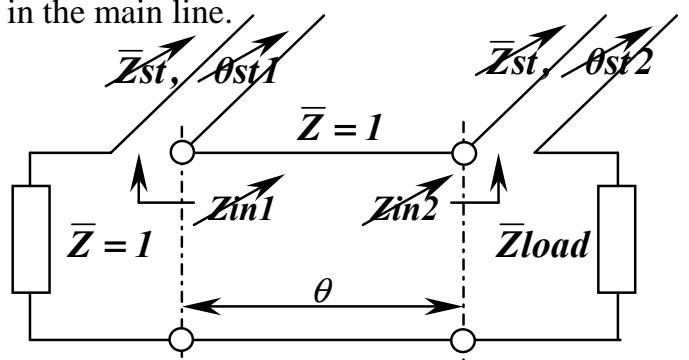

a)

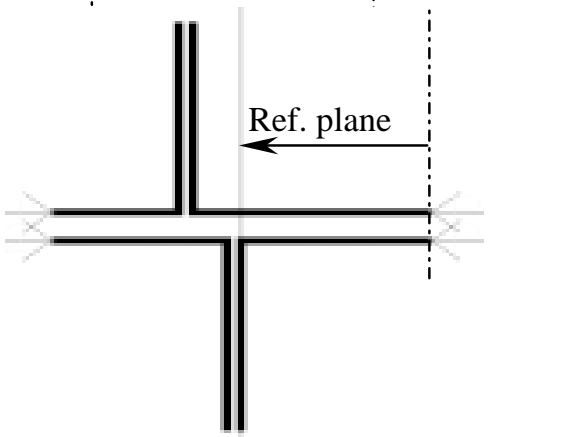

Fig.5 Double stub tuning circuit, for matching $\overline{Z l o a d}=1 \pm 0.1(1+j)(\mathrm{a})$, and its layout (b).

The layout of the matching circuit is shown in Fig.5b corresponding to matching $\bar{Z}$ load $=\mathbf{1 . 1}-\mathbf{j 0 . 1}$ and is characterized by electrically longest stubs $\theta 1$ and $\theta 2$ (they will be shortened under DC bias). A reference plane of the second port with impedance $(\overline{\boldsymbol{Z}} \boldsymbol{l o a d}=\mathbf{1 . 1}-\mathbf{j 0 . 1})$ is shifted as it is shown by a dashed line to provide a correct position for termination.

\subsection{Measurement}



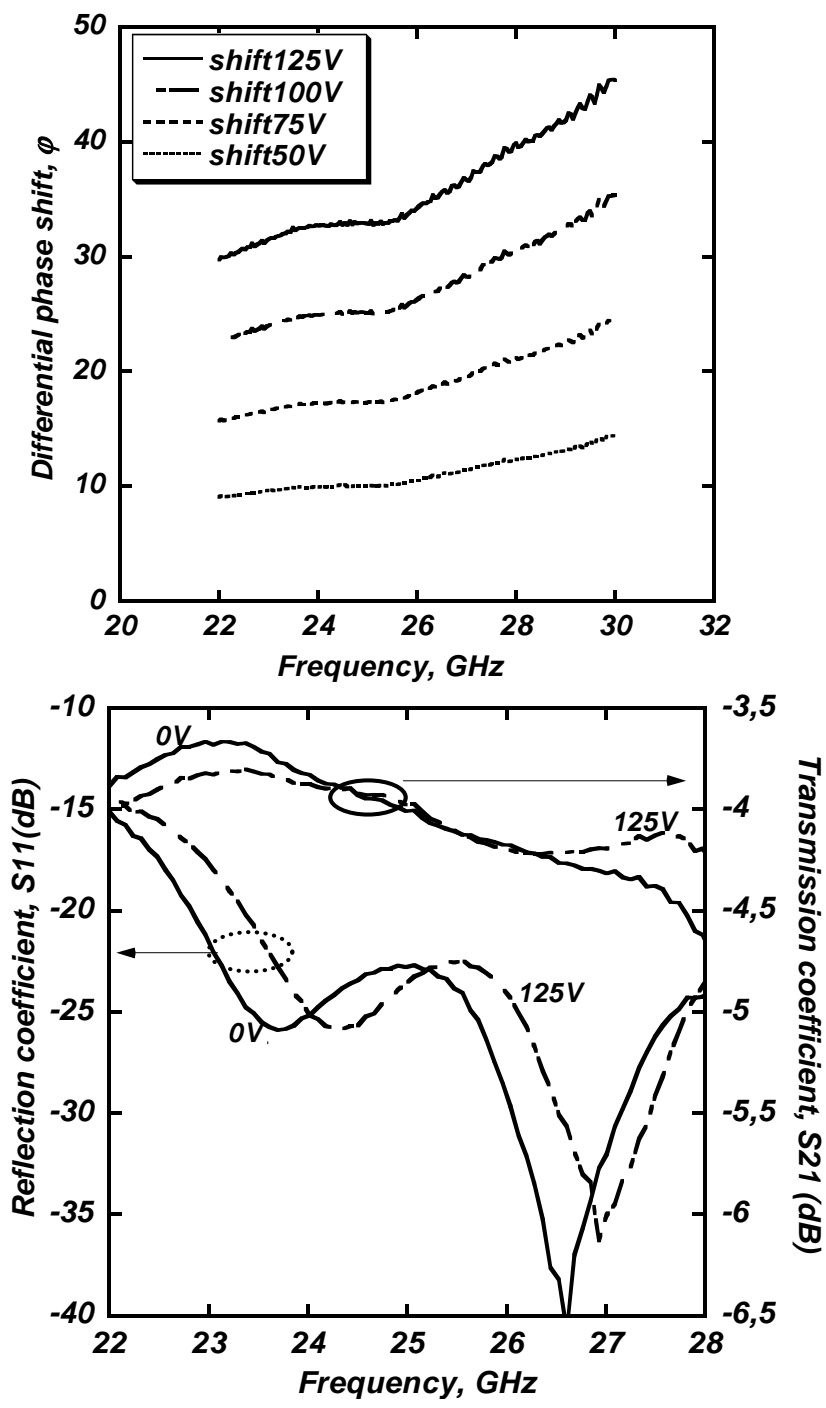

Fig.6. Measured phase-shifter performance.

Measured performance of the phase shifter is plotted in Fig.6. It should be noted that matching and transmission losses are in good agreement with simulated data, while obtained phase shift is significantly lower (33deg.) as compared to expected value (90deg.), which is due to the inhomogeneous DC field distribution in the ferroelectric film (see below). A rather week tunability was obtained experimentally for the matching network.

\section{Planar Versus Parallel Plate ELECTRODES}

Here we use a simple approximation to evaluate inhomogeneous distribution of field/permittivity. It is based on sequent use of standard SDA technique and stationary formula for static capacitance. In the first step we analyze transmission line problem in the static limit (i.e we define per unit length capacitance of the transmission line) using standard SDA formulation. This formulation is very accurate, since it involves rigorous representation of the Green function in the Fourier transformed domain. In the next step, the cross section of the transmission line is discretized and electric field within each cell is defined as function of applied to electrodes DC voltage. The permittivity within each cell is than modified using its DC field dependence. It is clear that inhomogeneous

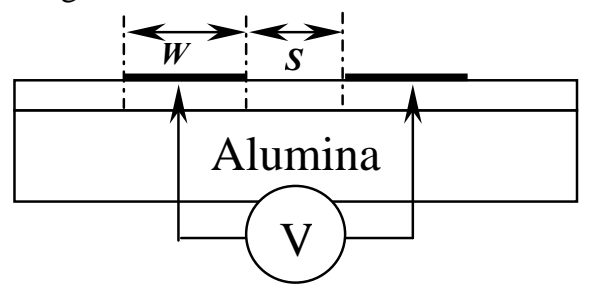

Fig.7 Planar transmission line problem for study of the tunability reduction effect.

distribution of permittivity (instead of uniform used in simulations) will deform the distribution of electric field. Using a stationary formula for capacitance we can expect that initial, although not accurate field distribution, will give rise to only second order error in per unit length capacitance of the transmission line under applied voltage. Hence, we can define the line tunability as follows:

$$
n=\frac{C(1)-C(V)}{C(1)}
$$

with per unit length capacitances defined as:

$$
C(V)=\frac{1}{V^{2}} \oint_{S} D(V) \cdot E(V) d s
$$

The integration is taken over the cross-section of the transmission line, Fig.7.

In the first approach we assume a linear dependence of the dielectric constant versus applied DC electric field. It is not a good approximation, but it helps to set the lower limit for tunability reduction, since in the real situation the material tunability in small DC fields will be lower. The first case analyzed is two $60 \mu \mathrm{m}$ strips separated by $20 \mu \mathrm{m}$ gap (applied voltage is $125 \mathrm{~V}$ ), which actually resembles the mentioned above phase shifter, Fig.4b. Obtained field distribution is shown in Fig.8 (limited below $2 \mathrm{~V} / \mu \mathrm{m}$ ). The area with stronger field is seen as a white spot between electrodes indicates the size of effectively tuned area. The calculated reduction factor for the line tunability is $\approx 2.46$, which fits well with experimentally obtained value. Fig. 8 also gives information regarding desired electrode width. It is easy to see that only small portion of electrode is in contact with area of effectively tuned ferroelectric, thus further increase of it width will only lead to limited increase of tunability. The dependence of the line tunability reduction versus electrode width with fixed $20 \mu \mathrm{m}$ gap was simulated and is given in Fig.9. 


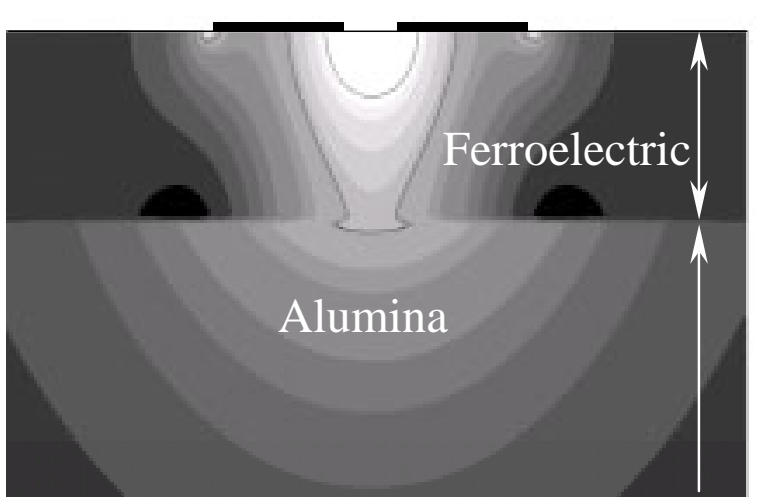

Fig.8 Picture of DC field in the coplanar strip line.

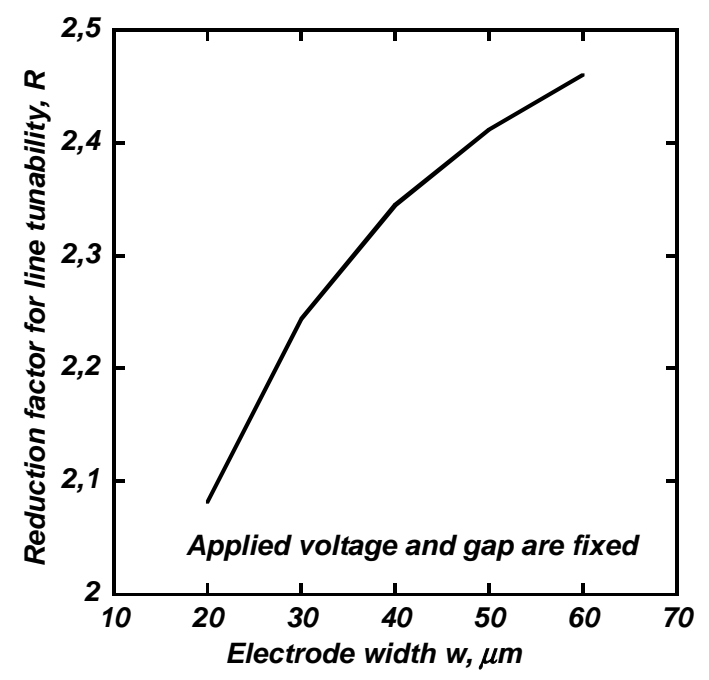

Fig.9 Simulated reduction of the transmission line tunability.

As it follows from the simulation no meaningful increase in line tunability can be achieved by decreasing the electrode width. The other possibilities are also limited by $20 \mu \mathrm{m}$ feature size limitation of gravure printing technique and $50 \mu \mathrm{m}$ limitation on ferroelectric layer thickness.

A significant increase in the line tunability can be achieved with a microstrip technology, which provides a possibility of parallel-plate tuning (DC field is essentially homogeneous).

\section{DESIGN AND PERFORMANCE OF DEVICES: PARALLEL-Plate ELECTRODES}

Design and experimental characteristics of two components using parallel-plate electrodes will be presented on poster session.

\section{CONCLUSION}

Possibilities of integration of ferroelectric films with tunable dialectic properties in LTCC are studied. The device demonstrators do not have adequate for systems applications performances, however they demonstrate the potentials of making adjustable/tunable components in LTCC technology, and pace a way for further materials and devices optimization. Currently work is in progress to develop new low loss high tunability ferroelectric compositions in LTCC processes for tunable microwave components.

\section{ACKNOWLEDGEMENTS}

The authors wish to acknowledge the European Commission for founding this project "MELODY" under Framework 5 and support of Swedish Science Foundation (VR). We would also like to thank Filtronic Comtek (UK) group for preparation of the basic BSTM powder.

\section{REFERENCES}

[1] H. Miyake, S. Kitazawa, T. Ishizaki, T. Yamada and Y. Nagatomi, "A miniaturized dual band filter using ceramic lamination technique for dual mode portable telephones," in IEEE MMT-S Int. Microwave Symp. Dig., vol 2, June 1997, pp. 789-792.

[2] A. Bailey, W. Foley, M. Hageman, C. Murray, A. Piloto, K. Sparks and K. Zaki, "Miniature LTCC filters for digital receivers," in IEEE MMT-S Int. Microwave Symp. Dig., vol. 2, June 1997, pp. 999-1002.

[3]T. Ishizaki, T. Yamada and H. Miyake, "A first practical model of very small and low insertion loss laminated duplexer using LTCC suitable for W-CDMA portable telephones," in IEEE MMT-S Int. Microwave Symp. Dig., vol. 1, June 2000, pp. 187-190.

[4] K. Kageyama, K. Saito, H. Murase, H. Utaki and T. Yamamoto, "Tunable active filter having multilayer structure using LTCC," IEEE Trans. Microwave Theory and Tech., vol. 49, 2001, pp. 2421-2424. 